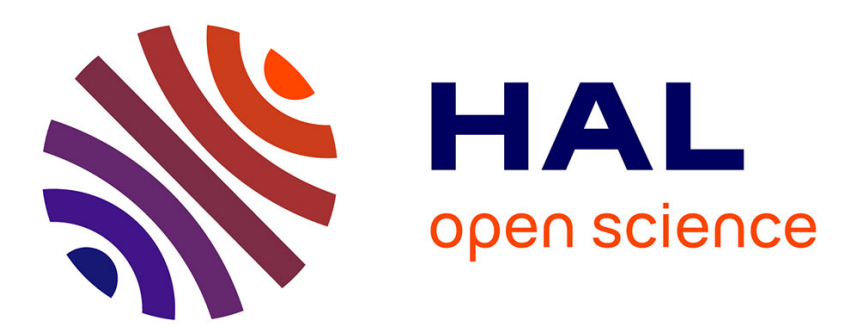

\title{
Aux limites de l'agriculture: les archives sédimentaires de la colonisation médiévale au Groenland
}

\author{
Vincent Bichet, Emilie Gauthier, Charly Massa, Christophe Petit, Hervé
}

Richard

\section{- To cite this version:}

Vincent Bichet, Emilie Gauthier, Charly Massa, Christophe Petit, Hervé Richard. Aux limites de l'agriculture: les archives sédimentaires de la colonisation médiévale au Groenland. J.F. Berger ed. Des climats et des hommes, Chapitre 18, Coédition La Découverte-Inrap, pp.307-325, 2012. hal02860082

\section{HAL Id: hal-02860082 \\ https://hal.science/hal-02860082}

Submitted on 8 Jun 2020

HAL is a multi-disciplinary open access archive for the deposit and dissemination of scientific research documents, whether they are published or not. The documents may come from teaching and research institutions in France or abroad, or from public or private research centers.
L'archive ouverte pluridisciplinaire HAL, est destinée au dépôt et à la diffusion de documents scientifiques de niveau recherche, publiés ou non, émanant des établissements d'enseignement et de recherche français ou étrangers, des laboratoires publics ou privés. 


\section{8 \\ Aux limites de l'agriculture : \\ les archives sédimentaires \\ de la colonisation médiévale au Groenland ${ }^{1}$}

Vincent Bichet ${ }^{*}$ Émilie Gauthier*, Charly Massa*, Christophe Petit ${ }^{\star \star}$, Hervé Richard ${ }^{\star}$

$\grave{A}$

la fin du vilie siècle, sous l'impulsion de différents facteurs dont la forte pression démographique qui pèse alors sur l'étroit littoral norvégien et l'essor du commerce scandinave, un vaste mouvement d'émigration en direction des régions subarctiques et arctiques s'amorce pour culminer, à la fin du $\mathrm{x}^{\mathrm{r}}$ siècle, avec la découverte du Groenland et des côtes de l'actuel Canada. De nombreux historiens s'accordent à dire que l'expansion viking vers les îles de l'Atlantique nord fût favorisée par l'embellie climatique du IX' au XII siècle [Lassen et al., 2004 ; Boyer, 2008], dénommée Optimum climatique médiéval. L'expansion se fit par étapes successives : les Scandinaves colonisent l'archipel des Orcades vers 800 , les Féroé vers 860 , puis l'Islande entre 874 et 930 . À la fin du Xe siècle, l'Islande, qui compte plus de 50000 habitants, connaît une sévère famine [Jones, 1987]. Ce contexte tourmenté ouvre la voie à une nouvelle migration et à la

- CNRS-université de Franche-Comté, UMR 6249, Laboratoire chrono-environnement.

** CNRS-université Paris-1 Panthéon-Sorbonne, UMR 7041, archéologle et sciences de l'Antiquité.

1 Ce projet de recherche bénéficie du soutien financier des universités de FrancheComté et de Bourgogne, du CNRS et l'Institut Paul-Émile Victor. 
colonisation du sud-ouest du Groenland, menée en l'an 986 par Eiríkr Thorvaldson, plus connu sous le nom d'Erik le Rouge.

$\mathrm{Au}$ Groenland, les émigrants fondent deux colonies principales à l'extrémité amont de fjords abrités : l'Østerbygden (Établissement oriental, $61^{\circ} \mathrm{N}$, région de Qaqortoq) qui se développa jusqu'à compter une cathédrale, une douzaine d'églises et de 190 à 220 fermes de dimensions variées [Jones, 1987], et le Vesterbygden (Établissement occidental) qui se développa à plus de $500 \mathrm{~km}$ au nord-ouest du premier $\left(64^{\circ} \mathrm{N}\right.$, région de Nuuk), comptant plus modestement 90 fermes et 4 églises.

L'économie des colonies vikings est fondée sur le commerce avec l'Islande et l'Europe et sur la généralisation, dans les limites des conditions du milieu, des pratiques agropastorales de l'époque, en particulier l'élevage bovin et ovin. Au maximum de leur développement, les colonies groenlandaises comptèrent, selon les estimations, entre 2250 [Lynnerup, 1996] et 6000 habitants [McGovern, 1991].

La chronologie de l'abandon des implantations vikings du Groenland, probablement vers 1350 pour l'établissement le plus au nord (Vesterbygden), et vers 1450 pour la colonie la plus méridionale (Østerbygden), est moins précise que celle de leur création. Les causes apparaissent multifactorielles et discutées [Barlow et al., 1997 ; Diamond, 2005]. Néanmoins, tous les auteurs s'accordent sur le rôle primordial de la péjoration climatique du Petit Âge glaciaire [Dugmore et al., 2007], qui succède à l'Optimum médiéval, et sur l'incapacité des populations vikings à s'adapter efficacement à l'évolution des conditions climatiques. Le froid et l'augmentation de l'occurrence des tempêtes provoquèrent sans doute une chute des rendements agricoles, isolèrent les colonies de l'Europe et coupèrent la route maritime du bois du Labrador. Est invoqué également le rôle de la montée du niveau océanique et la réduction corrélative de la surface des terres cultivables sur le littoral des fjords, provoquée par la subsidence progressive du continent sous le poids des glaciers en progression depuis la néoglaciation amorcée dès le $l^{\text {er }}$ millénaire avant notre ère [Mikkelsen et al., 2008].

Les travaux archéologiques et archéozoologiques menés depuis deux décennies attestent une tentative infructueuse d'adaptation des populations à l'émergence de la péjoration climatique. La réduction des troupeaux de bovins au profit des ovins, puis des caprins, moins exigeants en fourrage hivernal, accompagne en effet une sollicitation 
accrue des ressources cynégétiques terrestres ou marines durant la seconde moitié de la période de colonisation [Lynnerup, 1995 ; Arneborg et al., 1999 ; Enghoff, 2003].

En complément des effets de la dégradation climatique du Petit Âge glaciaire, des travaux récurrents mais peu soutenus par des arguments sédimentologiques irréfutables, défendent la théorie d'un effondrement de la colonie en raison d'une érosion catastrophique des sols provoquée par le développement excessif du pâturage [McGovern, 1991 ; Jakobsen, 1991 ; Dugmore et al., 2005 ; Mainland, 2006 ; Edwards et al., 2008].

Malgré les incertitudes qui pèsent sur la chronologie des événements et sur les modalités de subsistance des populations face aux aléas climatiques, l'étude de la colonisation viking du Groenland offre l'opportunité d'un modèle exceptionnel pour la compréhension des mécanismes d'interaction entre les sociétés, le climat et l'environnement. L'emprise agro-pastorale, le développement de la colonie, puis la déprise en moins de cinq siècles permettent en outre d'évaluer les conséquences environnementales de la pression anthropique sur un espace initialement naturel, puis la résilience de celui-ci après l'abandon des pratiques humaines invasives.

Diverses études paléoenvironnementales récentes [Schofield et al., 2007 ; 2008 ; Edwards et al., 2008 ; Buckland et al., 2009] ont abordé ce thème par le biais de l'analyse sédimentaire de séquences de sols archéologiques, de remplissages de fossés ou de dépôts tourbeux situés à proximité immédiate de sites archéologiques. Ces études ont permis d'améliorer l'acuité des connaissances mais se fondent sur des séquences trop souvent discontinues où les hiatus limitent une analyse synchrone de la totalité de la période d'occupation viking.

Pour pallier ces difficultés, nous avons entrepris d'étudier les archives sédimentaires lacustres disponibles au cœur des zones archéologiques [Massa et al., 2011]. L'étude des dépôts profonds des lacs permet en effet l'accès à une archive sensible aux variations environnementales et climatiques des bassins versants associés, offrant l'avantage d'une sédimentation continue et non perturbée couvrant potentiellement des durées plurimillénaires.

Un carottage a été réalisé dans la zone la plus profonde du lac $d^{\prime}$ 'galiku $\left(61^{\circ} 00^{\prime} 24^{\prime \prime} \mathrm{N}-45^{\circ} 26^{\prime} 30^{\prime \prime} \mathrm{W}\right)$, à proximité immédiate des vestiges archéologiques de Garðar, centre politique et religieux de 


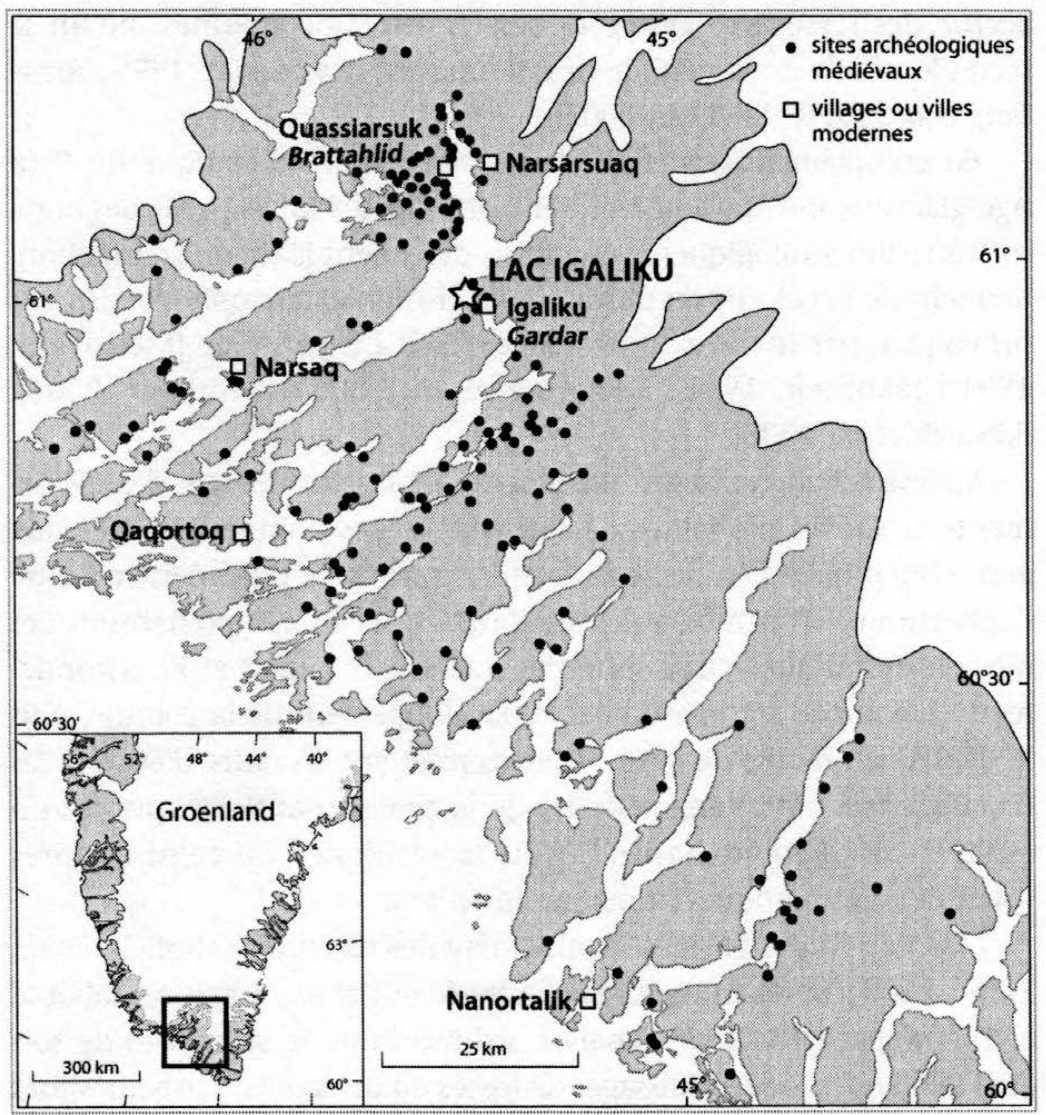

Figure 1 : Carte archéologique de l'Établissement oriental et localisation du lac d'Igaliku. 
l'Établissement oriental (fig. 1). Les premiers résultats obtenus à partir de cette séquence sédimentaire, en particulier l'analyse palynologique, offrent une lecture détaillée de la période viking et des contraintes environnementales de l'époque médiévale.

\section{Le contexte et les méthodes d'analyse}

Le lac d'Igaliku est un lac de 35 hectares situé au cœur d'un bassin versant de $\mathbf{4} \mathbf{k m}^{2}$, limité à l'est et à l'ouest par les fjords d'lgaliku et de Tunulliarfik, et par des reliefs avoisinant $400 \mathrm{~m}$ d'altitude au nord, et plus de $800 \mathrm{~m}$ au sud. Autour du lac, la végétation arbustive actuelle est dominée par le saule (Salix glauca), le bouleau (Betula glandulosa) et le genévrier (Juniperus communis). Une ferme associée à un parcellaire de prairies de fauche occupe aujourd'hui la rive sud du lac. Une très large part du bassin versant est pâturée par les moutons en période estivale, favorisant le développement d'une flore typique des milieux agropastoraux groenlandais. Les espèces les plus emblématiques de cette flore sont les renoncules (Ranunculus acris), l'oseille (Rumex acetosa) et le pissenlit (Taraxacum officinale).

Le lac ne reçoit aucun tributaire important ; les apports hydriques et sédimentaires sont essentiellement des apports diffus ou très localisés. Le carottage a été réalisé, au moyen d'une barge, au centre du lac, dans sa partie la plus profonde $(-20 \mathrm{~m})$. Une séquence de $4 \mathrm{~m}$ de longueur depuis l'interface eau-sédiment a été prélevée à l'aide d'un carottier gravitaire.

La chronologie de la séquence est établie par 28 dates carbone 14 dont 16 pour la période historique (fig. 2), réalisées sur des matières organiques terrestres, principalement des feuilles et des bois de bouleau et de saule, complétées pour la partie la plus récente, par la mesure de l'activité en radio-isotopes du plomb 210 et du césium 137.

Un vaste panel d'analyses sédimentologiques a été engagé sur cette séquence dans la perspective de déterminer les changements environnementaux survenus au cours des dix millénaires que représente la séquence sédimentaire. Une première série d'analyses diagraphiques a été réalisée sur la carotte non déstructurée, avec un pas de mesure millimétrique, soit une résolution moyenne de 2,5 ans par point d'analyse. Ces mesures correspondent à des analyses pétrophysiques ( $\gamma$-densité, imagerie-RX, susceptibilité magnétique, spectrocolorimétrie) et 

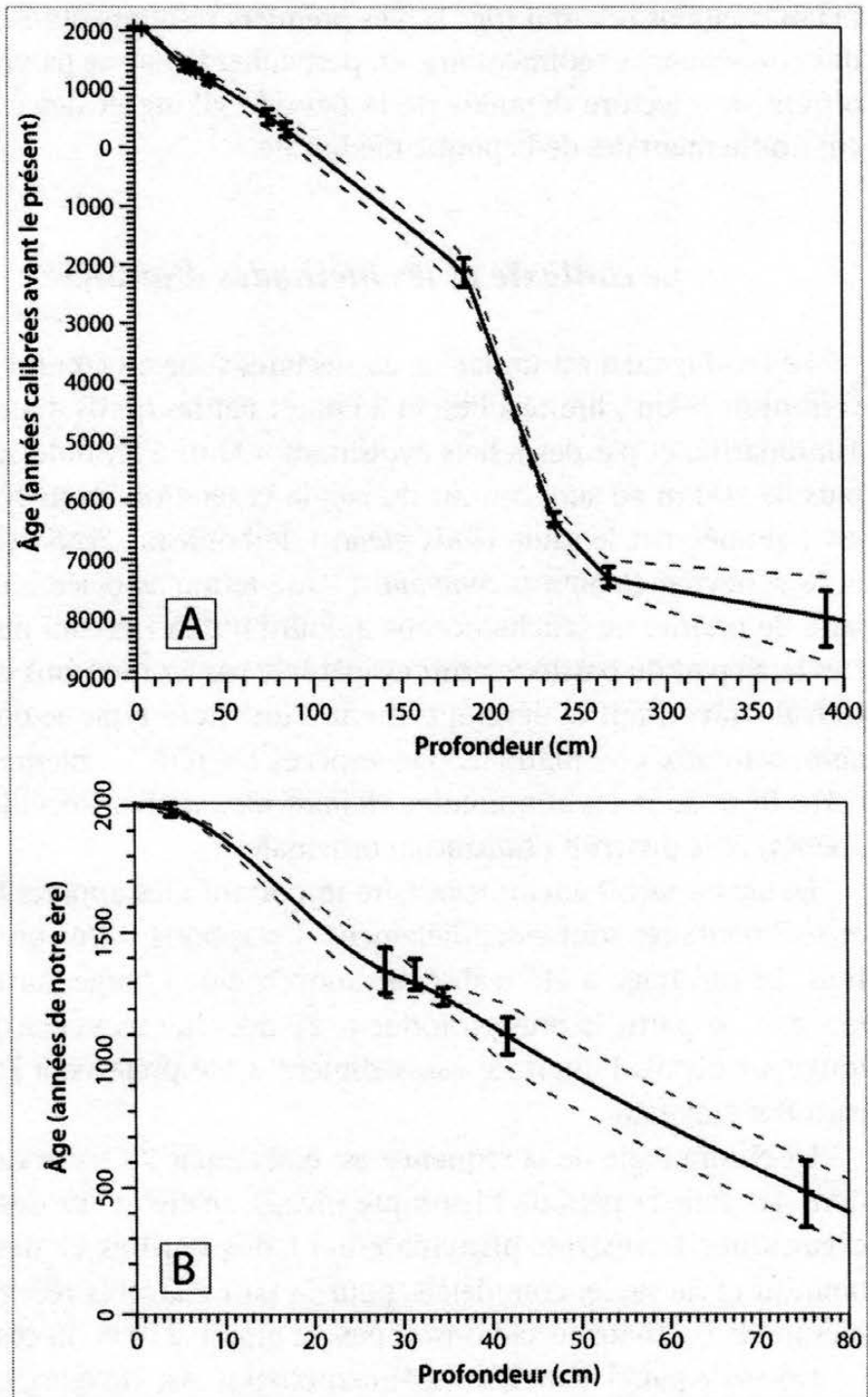

Figure 2 : (A) Stratigraphie et modèle âge-profondeur de la séquence du lac d'Igaliku ; (B) Détail pour la période historique. 
géochimiques (analyse élémentaire XRF). Elles sont complétées par une série d'analyses sur échantillons, avec une résolution de l'ordre de 20 ans pour la période historique et de 50 ans pour le reste de la séquence. La démarche analytique porte sur les paramètres biotiques (pollens, spores et microfossiles non polliniques, diatomées, chironomes, géochimie organique) et abiotiques ( $\mu$-granulométrie, minéralogie, géochimie des éléments majeurs et ETM, géochimie isotopique $\mathrm{N}-\mathrm{C})$.

Pour les 1500 dernières années, dans la perspective de discriminer l'enregistrement des effets de l'occupation médiévale, l'analyse quantitative de la fraction minérale du sédiment associée à la chronologie des dépôts, permet le calcul d'un taux d'érosion moyen des sols du bassin versant du lac (modalités de calcul détaillées dans Massa et al., à paraître).

Concernant l'analyse pollinique dont les résultats sont plus particulièrement développés ici [Gauthier et al., 2010], un minimum de 400 grains de pollen de plantes terrestres a été déterminé dans chaque échantillon. L'étude des grains de pollen est complétée par la détermination et le comptage des microfossiles non polliniques (spores de champignons, cyanobactéries, algues, petits invertébrés...). Dans cette étude, une attention particulière a été portée aux spores de champignons coprophiles (Sordaria, Sporormiella, Podospora, Amium...) dont la présence dans les sédiments lacustres est associée aux excréments d'herbivores, et plus particulièrement de bétail domestique, qui pâturent dans le bassin versant pendant les périodes d'activité agropastorale, ou encore aux épandages de fumier qui ont pu être pratiqués par les Vikings pour améliorer la fertilité des sols [Commisso et Nelson, 2008 ; Buckland et al., 2009].

\section{Résultats et discussion}

La séquence sédimentaire du lac d'Igaliku présente une stratigraphie contrastée qui peut être distinguée en deux unités principales : à la base $(-400$ à $-275 \mathrm{~cm})$, un ensemble de silts et silts-sableux gris, sans figure sédimentaire apparente, associé à de nombreux graviers et cailloux ; dans la partie supérieure $(-275 \mathrm{~cm}$ à $0 \mathrm{~cm})$, une unité siltosableuse laminée, à fraction organique relativement abondante. Le modèle âge-profondeur (fig. $2 a$ ) atteste d'une sédimentation continue 


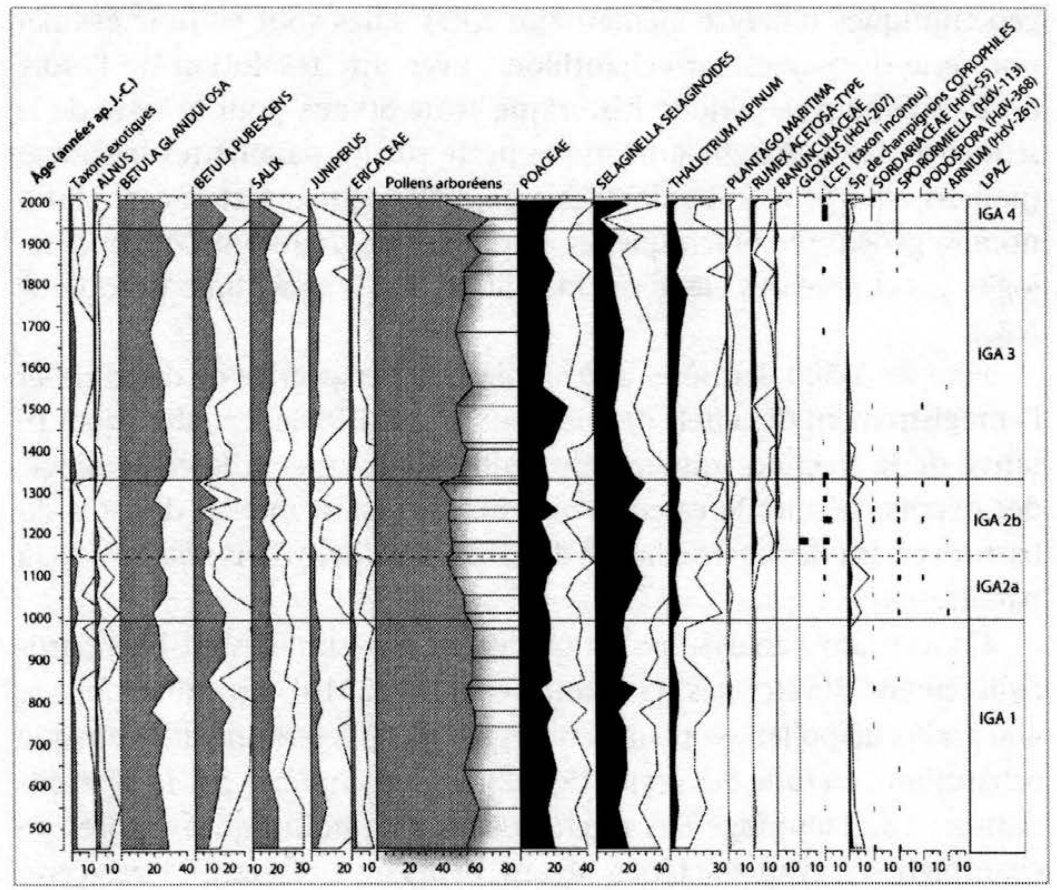

Figure 3 : Diagramme palynologique simplifié (pollens et microfossiles non polliniques) en valeurs relatives. Courbes d'exagération x 2 pour Alnus, Betula pubescens, Salix, Juniperus, Ericaceae, Poaceae, Selaginella selaginoïdes; x 5 pour Exotic taxa, Thalictrum, Plantago, Rumex, Ranunculaceae et la somme des spores de champignons coprophiles. La courbe des taxons exotiques cumule les pourcentages de : Abies, Picea, Pinus, Carpinus, Corylus, Fagus, Fraxinus, Quercus, Ulmus, Ambrosia et Artemisia. 
dont la chronologie couvre les dix derniers millénaires, soit la quasitotalité de l'Holocène. L'unité inférieure correspond à une sédimentation en contexte glaciogénique et traduit un taux de sédimentation rapide, de l'ordre de 2,3 mm par an. La chronologie de l'unité supérieure laminée débute aux environs de 7550 avant notre ère, et le taux de sédimentation varie de $0,2 \mathrm{~mm}$ par an entre 7550 et 3050 avant notre ère, à $0,41 \mathrm{~mm}$ par an entre -3050 et aujourd'hui. Au cours de la période médiévale, les XI et XIIr siècles se distinguent par une accélération du taux de sédimentation qui atteint $0,85 \mathrm{~mm}$ par an, soit plus du double de la valeur correspondante au taux moyen de la période historique (fig. $2 b$ ).

\section{Changements de végétation et marqueurs du pastoralisme}

L'analyse palynologique couplée à l'étude des microfossiles non polliniques des 1500 dernières années (fig. 3) révèle avec une grande lisibilité l'évolution du couvert végétal et les phases d'emprise et de déprise agropastorales.

La première partie du diagramme synthétique (zone pollinique locale IGA 1, fig. 4) correspondant à la seconde moitié du $1^{\mathrm{er}}$ millénaire, révèle un spectre végétal caractéristique des milieux naturels de la région subarctique : les taxons arboréens et les arbustes (Betula pubescens et glandulosa, Salix, Juniperus et Ericaceae) dominent le spectre et représentent près de $60 \%$ de la somme pollinique. Cette végétation permet le développement de la strate herbacée (Poaceae) et des mousses (Selaginella selaginoïdes). Cette zone ne comporte aucun indice de pratique agricole et les pourcentages de spores de champignons coprophiles sont insuffisants pour traduire une quelconque pression pastorale. Les rares grains de pollen de Rumex acetosa type (oseille) présents à ce niveau, peuvent être considérés comme issus de Polygonaceae autochtones comme Oxyria dygina, ou de variétés allochtones, telles que Rumex acetosella qui est indigène plus au nord du Groenland (aux environs de $71^{\circ} \mathrm{N}$ sur la côte ouest, et entre $65^{\circ}$ et $70^{\circ} \mathrm{N}$ sur la côte est). La présence de ce taxon peut aussi être associée à des transports aériens de pollens en provenance d'lslande ou d'Amérique (d'autres taxons « exotiques »-Picea, Abies, Pinus, Ulmus, Ambrosia... - confirment l'existence de ces apports lointains).

La composition relative du spectre pollinique marque une évolution vers l'an mille (zone pollinique IGA 2, fig. 3) qui traduit le passage 


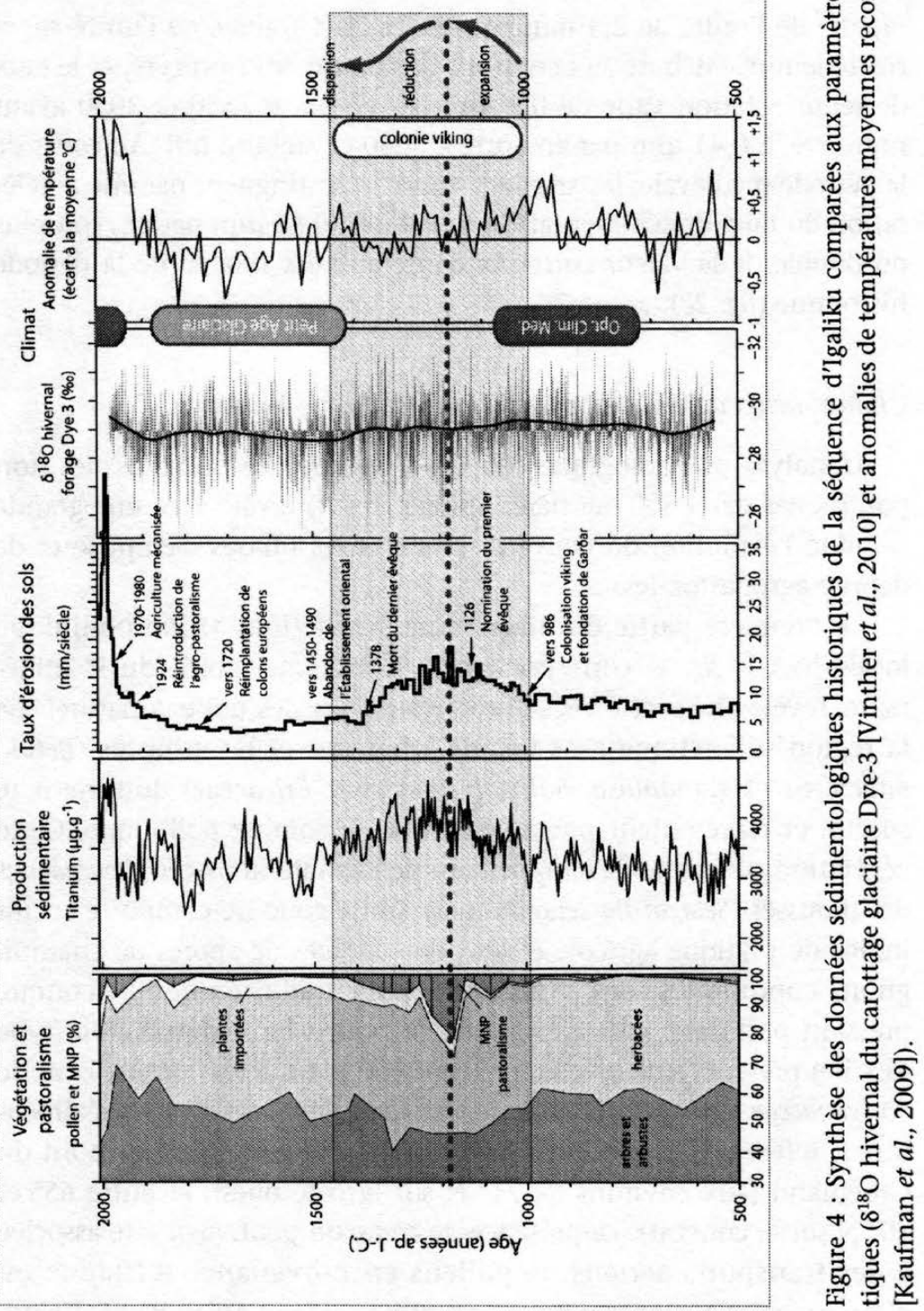


d'un milieu naturel à un milieu anthropisé. Cette transition intervient au cours de la zone $2 \mathrm{a}$, qui débute un peu avant l'an mille et se poursuit jusque vers 1150 environ. Au cours de cette période, les premiers changements environnementaux sont discrets mais indéniables. On constate une réduction générale du couvert arboré (le taux de pollen d'arbres passe de $60 \%$ à environ $40 \%$ ) au profit de l'augmentation des herbacées et bryophytes héliophiles comme Thalictrum et Selaginella. Parmi les taxons arboréens, le bouleau est le plus affecté et la diminution plus drastique de Betula pubescens que de Betula glandulosa indique une réduction sélective des bois de plus grande taille. Parallèlement, les grains de pollen de bouleau corrodés sont nombreux et associés à une augmentation du taux de chlamydospores de Glomus, champignon parasite des racines d'une large variété de plantes, en particulier de Betula [van Geel, 2001], dont la présence signe une probable érosion des sols [Lawson et al., 2007].

Cette ouverture de la toundra subarctique s'accompagne d'une nette augmentation des valeurs des spores de champignons coprophiles significatifs de la présence d'herbivores dans le bassin versant [van Geel et Aptroot, 2006; Davis et Shafer, 2006]. Ces évolutions de la végétation, synchrones de la colonisation et de la fondation de $\mu$, constituent une réponse directe à l'implantation viking, au développement de pratiques agropastorales (défrichement, pâturage du bétail) et probablement à l'exploitation des "grands » arbres pour les usages domestiques (bois de chauffe, constructions).

Pour autant, les plantes rudérales en particulier Rumex acetosa type - l'un des principaux marqueurs polliniques de l'anthropisation dans le sud du Groenland, dont le développement lors de la colonisation médiévale est considéré comme invasif et probablement associé à l'expansion de plants accidentellement importés [Fredskild, 1973; $1978 ; 1992$ ] - sont très peu présentes au cours de cette première phase anthropisée. Est-ce la traduction d'un impact anthropique limité et d'une colonie en développement? Probablement, puisque les textes historiques relatent que le peuplement de la région d'lgaliku se développa dès l'arrivée d'Erik le Rouge mais qu'il ne prit son réel essor qu'à l'installation de l'évêché de Garðar vers 1126 [Buckland et al., 2009]. Dans le diagramme pollinique d'Igaliku, c'est précisément vers 1100 que les pourcentages de Rumex acetosa type commencent à augmenter.

À compter de 1150 (zone pollinique $2 b, f i g .3$ ) les marqueurs polliniques et non polliniques de l'anthropisation sont clairement 
exprimés : apophytes indigènes et non indigènes (Rumex acetosa type, Ranunculus acris type...), spores de champignons coprophiles (Sporormiella, Sordaria, Arnium...). Ces dernières traduisent la pression pastorale qui s'exerce dans le bassin versant mais peut-être également l'épandage de fumier dans les prairies à foin.

Pour autant, cet état d'anthropisation du bassin versant du lac ne dure pas : à partir de 1320 environ (début de la zone pollinique IGA 3, fig. 3), l'augmentation de grains de pollen non corrodés, la diminution des spores de champignons coprophiles et l'augmentation du taux de pollens arboréens suggèrent alors une très nette réduction, voire l'abandon de l'activité pastorale. Ce constat confirme l'hypothèse d'un affaiblissement de la productivité agricole vers la fin du XIII" siècle déjà proposée par Arneborg et al. [1999; 2002]. Ces travaux ont en effet démontré que le régime alimentaire des Vikings avait évolué entre le $x^{e}$ et le $x v^{e}$ siècle, passant progressivement d'une alimentation à $80 \%$ d'origine terrestre jusqu'au XIII siècle, à une alimentation majoritairement d'origine marine au cours du xIII siècle et jusqu'à l'abandon, quand les aliments d'origine terrestre ne représentent plus que $20 \%$ des ressources des populations.

En moins d'un siècle à partir de 1320 , l'ensemble des marqueurs signe la totale disparition des activités agropastorales à proximité du lac. Pour autant, la résilience de l'écosystème végétal, après l'abandon de la colonie viking (datée vers 1450-1490 par les textes et l'archéologie), paraît incomplète puisque des taxons favorisés par l'exploitation agricole viking, comme Rumex acetosa en particulier, demeurent présents et le restent encore aujourd'hui. La végétation locale est durablement marquée par l'empreinte de l'agropastoralisme viking.

Avec le retour des colons danois au XviII siècle, qui établissent le village d'lgaliku sur les ruines de Garðar, mais surtout avec le renouveau de l'agriculture groenlandaise à partir de 1920, les indices polliniques d'anthropisation marquent une reprise de l'agropastoralisme avec des effets sur la végétation comparables à ceux enregistrés durant la période d'occupation viking.

\section{L'érosion des sols}

Si des indices d'érosion des sols sont mis en évidence par l'analyse des grains de pollen et des microfossiles non polliniques, ils sont insuffisants pour quantifier la variabilité de cette érosion au cours des 
1500 dernières années. L'analyse des paramètres abiotiques, en particulier l'évaluation de la teneur en matière minérale et en titanium de la séquence sédimentaire, offrent l'opportunité de remédier à cette insuffisance (fig. 4). Le titanium est un élément géogène conservatif contenu dans les sols du bassins versant, dont la variabilité dans les dépôts lacustres est significative de l'érosion des sols [Kylander et al., 2011 ; Massa et al., à paraître]. Au cours de la seconde moitié du $\mathrm{I}^{\mathrm{er}}$ millénaire, avant la colonisation, la teneur en titanium des sédiments du lac d'Igaliku montre une variabilité à haute fréquence dont la valeur médiane est cependant assez constante et légèrement supérieure à $3000 \mu \mathrm{g} \cdot \mathrm{g}^{-1}$. Cette valeur augmente à partir de l'an mille pour atteindre $3500 \mu \mathrm{g} \cdot \mathrm{g}^{-1}$ durant le XI siècle et $4000 \mu \mathrm{g} \cdot \mathrm{g}^{-1}$ durant les XII et XIII siècles. La teneur en titanium est ensuite décroissante au cours du XIVe siècle et retourne à des taux médians de $3000 \mu \mathrm{g} \cdot \mathrm{g}^{-1}$ durant les siècles suivants. Parallèlement, le taux d'érosion des sols (fig. 4), calculé à partir du modèle âge-profondeur, du taux de matière minérale sédimentée et des densité et surface des sols du bassin versant [Massa et al., à paraître], suit globalement la même tendance. Durant la seconde moitié du ler $^{\text {er }}$ millénaire, avant l'arrivée des colons vikings, le taux d'érosion naturel des sols est relativement constant, entre 5 et $7 \mathrm{~mm}$ par siècle. Dès l'an mille, et de façon synchrone à l'arrivée des colons, le taux d'érosion augmente pour atteindre son maximum, $15 \mathrm{~mm}$ par siècle (soit plus de 2 fois la valeur antérieure) vers 1150-1170. À compter de cette période, la courbe est décroissante et le taux d'érosion retrouve, avant la fin du XIV siècle, des valeurs similaires au taux d'érosion en conditions naturelles, c'est-à-dire voisine de $5 \mathrm{~mm} /$ siècle. Une fois encore, à travers la courbe du taux d'érosion, la période médiévale se singularise très nettement (fig. 4). La longue période de quatre siècles du Petit Âge glaciaire qui succède à l'époque médiévale, se traduit quant à elle par une érosion très faible, probablement limitée par une forte réduction des processus hydriques. Il faut attendre le retour des agriculteurs au XIXe siècle pour voir à nouveau croître l'érosion des sols. L'introduction de l'agriculture mécanisée et le terrassement des prairies à foins, au début des années 1880 , entraînent une érosion exceptionnelle de près de $40 \mathrm{~mm}$ par siècle, sans équivalent au cours de la période historique. 
L'enregistrement sédimentaire historique du lac d'Igaliku, à travers le spectre pollinique de la végétation et la production minérale du bassin versant, révèle des changements environnementaux peu corrélés avec la variabilité climatique mesurée au cours de la même période (fig. 4). Les courbes climatiques disponibles pour cette région arctique, qu'il s'agisse de la variabilité des températures hivernales estimées à partir du $\delta^{18} \mathrm{O}$ du carottage glaciaire de Dye-3 [Vinther $e t$ al., 2010] ou des anomalies des températures moyennes annuelles reconstituées [Kaufman et al., 2009], indiquent des changements importants mais peu incidents sur la dynamique de l'écosystème lacustre. Cette situation, a priori paradoxale, est sans doute due à la localisation géographique du lac, toujours situé à moins de $10 \mathrm{~km}$ de l'inlandsis groenlandais, quelle que soit la dynamique climatique. La réponse sédimentaire aux changements du climat, dans ces conditions, est très largement tamponnée. En revanche ce contexte met en évidence de façon optimale la réponse sédimentaire liée aux activités humaines, dont il faut tout de même admettre qu'elles sont, dans cette région et durant la période historique, largement sous le contrôle des changements climatiques.

La chronologie des événements mise en évidence par cette étude montre en effet que les colons scandinaves s'implantent au Groenland au maximum de l'Optimum climatique médiéval, daté vers l'an mille dans cette région du monde [Vinther et al., 2010]. À compter de cette époque, la tendance longue du climat est au refroidissement progressif. Pour autant, cela ne semble pas préjudiciable, dans un premier temps, au développement de la colonie qui déploie ses activités agropastorales et atteindra une taille suffisante pour qu'un évêque soit nommé en 1126 [Arneborg, 2007]. Vers la fin du XII' siècle, marqueurs du pastoralisme et indices d'érosion sont au paroxysme. Mais la situation apparaît précaire puisque les données sédimentaires indiquent une rupture avant la fin du même siècle, traduisant une réduction progressive mais très nette de l'agropastoralisme autour du lac d'Igaliku, d'abord marqué par une réduction du taux d'érosion des sols puis, avec un retard de quelques décennies, par l'évolution de la végétation et une réduction des marqueurs du pastoralisme. Cette décroissance est irrémédiable puisqu'elle abouti à la quasi-disparition de l'activité agricole avant la fin du XIV' siècle, à la probable réduction 
démographique de la colonie (le dernier évêque connu meurt en 1378), puis à l'abandon définitif quelques décennies plus tard, en même temps que l'installation de la récurrence climatique froide du Petit Âge glaciaire. Quelles sont les causes de cette rupture qui entraîne la décroissance de la communauté viking à la fin du XII siècle ? Une succession de crises climatiques (hivers très rigoureux), mises en évidence à cette période par les archives glaciaires du forage Dye-3 [Vinther et al., 2010], pourrait peut-être avoir eu des incidences sur un démarrage tardif de la saison végétative estivale et engendré une chute des rendements agricoles suffisamment importante pour perturber durablement l'équilibre de la société viking.

\section{Conclusion et perspectives}

L'histoire du Groenland est celle de la survie et de l'adaptation des hommes aux conditions climatiques difficiles de l'Arctique, dans les limites de leurs traditions culturelles. Les particularités climatiques de la région et le contexte de sa colonisation ont engendré un système agraire fragile, proche de ses limites et sensible aux variations du climat. La brièveté de la colonisation médiévale, son caractère contrasté et la richesse des informations apportées à ce sujet par la communauté des archéologues font du modèle agropastoral scandinave du Groenland un cas d'étude exceptionnel dans le cadre des recherches paléoenvironnementales.

L'étude des sédiments lacustres, en particulier sur le site d'Igaliku, offre l'opportunité de revisiter à haute résolution temporelle l'histoire et les conséquences environnementales de la colonisation.

Si ces premiers résultats confirment la chronologie de l'occupation viking déjà définie par les textes historiques et l'archéologie, ils conduisent, en tous cas à Igaliku, à contredire la vision catastrophiste d'un abandon lié à la surexploitation du milieu défendue par certains auteurs [McGovern, 1991 ; Jakobsen, 1991 ; Dugmore et al., 2005 ; Edwards et al., 2008]. Les taux d'érosion des sols calculés pour l'époque médiévale sont modestes, compatibles avec des pratiques agricoles "durables" [Montgomery, 2007], et en tout cas sans commune mesure avec les incidences de l'agriculture moderne qui s'est récemment développée autour du lac. 
L'analyse sédimentaire traduit également un écosystème lacustre dont les changements apparaissent sous le contrôle dominant des activités anthropiques en particulier durant l'époque médiévale. L'agropastoralisme viking a marqué l'environnement d'une empreinte lisible et peu résiliente, sans pour autant avoir engendré une mutation écosystémique profonde, à la différence des activités agricoles contemporaines, appelées à se développer davantage dans le contexte du réchauffement climatique en cours, qui produiront sans doute des impacts environnementaux beaucoup plus spectaculaires.

L'étude des sédiments du lac d'Igaliku précise la chronologie et les modalités de l'expansion, durant 200 ans, de la colonie viking du sudouest groenlandais, puis son repli sur près de deux siècles également, à compter de la fin du xIr siècle. Les causes climatiques à l'origine du repli sont entrevues et devront être précisées dans le cadre de la poursuite des travaux.

Sur le plan méthodologique, il apparaît que l'étude des paramètres biotiques permet, dans ce contexte, une évaluation optimale des impacts anthropiques. Outre les pollens et les microfossiles non polliniques, les paramètres biologiques, biogéochimiques et isotopiques, relevant du fonctionnement trophique lacustre et sensibles aux activités agricoles (dispersion d'effluents d'élevage liée au pâturage et à l'amendement des sols dans le bassin versant du lac), sont un axe analytique à privilégier pour une lisibilité accrue des pratiques agricoles médiévales.

Enfin, pour étendre les observations réalisées à lgaliku à l'ensemble des secteurs de l'occupation médiévale, de nouveaux carottages ont été entrepris dans d'autres lacs, tant dans l'Établissement oriental (Østerbygden) que dans l'Établissement occidental (Vesterbygden), dans la région de Nuuk. Leur étude apportera sans doute une contribution à l'histoire de la société médiévale scandinave du Groenland, à l'extrême limite des capacités d'adaptation du modèle agropastoral européen.

Références bibliographiques

ARNeborg J., HeInemeier J., LynNerup N., Nielsen H. L., Rud N., SVEINBJÖRNSDÓTTIR Á. E. (1999), « Change of diet of the Greenland Vikings determined from stable carbon isotope analysis and $14 \mathrm{C}$ dating of their bones ", Radiocarbon, $n^{\circ} 41$, p. 157-168. 
ArNeborg J., Heinemeier J., LynNerup N., Nieisen H. L., Rud N., SVEINBJORNSDÓTTIR Á. E. (2002), « C-14 dating and the disappearance of Norsemen from Greenland », Europhysics News, p. 77-80.

ARNEBORG J. (2007), Saga trails. Brattahlid, Gardar, Hvalsey fiord's church and heriolfnesnes : four chieftain's farmsteads in the north settlements of Greenland. A visitor's guidebook, The National Museum of Denmark, Copenhagen.

Barlow L. K., Sadler J. P., Ogilvie A. E. J., Buckland P. C., Amorosi T., Ingimundarson J. H., Skidmore P., Dugmore A. J., McGovern T. H. (1997), " Interdisciplinary investigations of the end of the Norse Western Settlement in Greenland ", The Holocene, $n^{\circ} 7$, p. 489-499.

BOYER R. (2008), Les Vikings - Histoire, mythes, dictionnaire, Robert Laffond, Paris.

Buckland P. C., Edwards K. J., Panagiotakopulu E., Schofield J. E. (2009), « Palaeoecological and historical evidence for manuring and irrigation at Garthar (Igaliku), Norse Eastern Settlement, Greenland », The Holocene, $\mathrm{n}^{\circ}$ 19, p. 105-1 16.

Commisso R. G., Nelson D. E. (2008), « Correlation between modern plant [delta] $15 \mathrm{~N}$ values and activity areas of Medieval Norse farms $"$, Journal of Archaeological Science, $\mathrm{n}^{\circ}$ 35(2), p. 492-504.

Davis O. K., Shafer D. S. (2006), « Sporormiella fungal spores, a palynological means of detecting herbivore density ${ }_{\text {, }}$ Palaeogeography, Palaeoclimatology, Palaeoecology, $\mathrm{n}^{\circ}$ 237, p. 40-50. Diamond J. (2005), Collapse: How Societies Choose to Fail or Survive, Penguin/Allen Lane, Londres.

Dugmore A .J., Church M. J., Buckland P. C., Edwards K. J., Lawson I., McGovern T. H., Panagiotakopulu E., Simison l. A., Skidmore P., SveinbJaknardótTir G. (2005), * The Norse landnám on the North Atlantic islands : an environmental impact assessment ", Polar Record, $\mathrm{n}^{\circ}$ 41, p. 21-37.

Dugmore A., Borthwick D., ChurCh M., Dawson A., Edwards K., Keller C., Mayewski P., McGovern T., Mairs K. A., SveinbjarnardótTir G. (2007), « The Role of Climate in Settlement and Landscape Change in the North Atlantic Islands : An Assessment of Cumulative Deviations in High-Resolution Proxy Climate Records ", Human Ecology, n³ 35, p. 169-178.

Edwaros K. J., Schofield J. E., Mavquoy D. (2008), « ligh resolution paleoenvironmental and chronological investigations of Norse landnam at Tasiusaq, Eastern Settlement, Greenland », Quaternary Research, $\mathrm{n}^{\circ}$ 69, p. 1-15.

ENGHOFF 1. B. (2003), « Hunting, fishing and animal husbandry at the Farm Beneath the Sand, Western Greenland. An 
archaeozoological analysis of a Norse farm in the Western Settlement ", Meddelelser om Gronland, Man and Society, $\mathrm{n}^{\circ} 28$.

FreDSKILD B. (1973), "Studies in the vegetational history of Greenland ", Meddelelser om Gronland, $\mathrm{n}^{\circ} 198$, p. 1-245.

FreDsKILD B. (1978), « Paleobotanical investigations of some peat deposits of Norse age at Qagissiarssuk, South Greenland ", Meddelelser om Gronland, $\mathrm{n}^{\circ}$ 204, p. 1-41.

FREDSKILD B. (1992), "Erosion and vegetational changes in South Greenland caused by agriculture », Geografisk Tidsskrift, $n^{\circ} 92$, p. 14-21.

Gauthier E., Bichet V., Massa C., Petit C., Vanniere B., Richard H. (2010), « Pollen and non-pollen palynomorph evidence of medieval farming activities in South Western Greenland $»$, Vegetation History and Archaeobotany, $\mathrm{n}^{\circ} 19$, p. 427-438.

JAKOBSEN B. H. (1991), « Soil resources and soil erosion in the Norse settlement area of Østerbygden in Southern Greenland », Acta Borealia, $\mathrm{n}^{\circ} 8$, p. 56-68.

JONEs G. (1987), A History of the Vikings, Oxford University Press, Oxford.

Kaufman D. S., Schneider D. P., McKay N. P., Ammann C. M., Bradley R. S., Briffa K. R., Miller G. H., Otto-Bliesner B. L., OverPeCK J. T., Vinther B. M., Members A. L.k.P. (2009), « Recent warming reverses long-term Arctic cooling ", Science, $\mathrm{n}^{\circ} 325$, p. 1236-1239.

Kylander M. E., Ampel L., Wohlfarth B., Veres D. (2011), * High-resolution X-ray fluorescence core scanning analysis of Les Echets (France) sedimentary sequence : new insights from chemical proxies », Joumal of Quaternary Science, $n^{\circ} 26$, p. 109-117.

LASSEN S. J., Kuijpers A., Kunzendorf H., HoffMANN-Wieck G., Mikkelsen N., KonRadi P. (2004), "Late-Holocene Atlantic bottom-water variability in Igaliku Fjord, South Greenland, reconstructed from foraminifera faunas $"$, The Holocene, $\mathrm{n}^{\circ} 14$, p. 165-171.

Lawson I. T., Gathorne-Hardy F., Church M. J., Newton A. J., Edwards K. J., Dugmore A. J., Enarsson A. (2007), « Environmental impacts of the Norse settlement : palaeoenvironmental data from Mvatnssveit, Northern lceland ", Boreas, $\mathrm{n}^{\circ} 36-1$, p. 1-19.

LYNNERUP N. (1995), The Greenland Norse : A Biological Anthropological Study, Thèse de doctorat non publiée, université de Copenhague. LYNNERUP N. (1996), "Paleodemography of the Greenland Norse ", Arctic Anthropology, $\mathrm{n}^{\circ} 33$, p. 122-136. 
MaInland l. (2006), « Pastures lost? A dental microwear study of ovicaprine diet and management in Norse Greenland », Journal of Archaeological Science, $n^{\circ} 33$, p. 238-252.

Massa C., Bichet V., Gauthier E., Petit C., Richard H. (2011) - « Vers l'Amérique : l'implantation médiévale scandinave de la côte sud-ouest du Groenland. Approches historiques et premiers résultats des études paléoenvironnementales ", in Peuplements et préhistoire en Amériques, éditions CTHS, p. 181-191.

Massa C., Bichet V., Gauthier E., Perren B. B., Mathieu O., Petit C., MonNa F., Giraudeau J., Losno R., Richard H. (à paraître), "A 2500 year record of natural and anthropogenic soil erosion in South Greenland », Quatemary Science Review.

MCGOVERN T. H. (1991), « Climate correlation and causation in Norse Greenland », Arctic Anthropology, n² 28, p. 77-100.

Mikkelsen N., Kuijpers A., ARneborG J. (2008), " The Norse in Greenland and Late Holocene sea-level change $»$, Polar Record, $n^{\circ} 44$, p. 45-50.

MONTGOMERY D. R. (2007), " Soil erosion and agricultural sustainability ", Proceedings of the National Academy of Sciences $\mathrm{n}^{\circ} 104$, p. 13268-13272.

SChofield J. E., Edwards K. J., McMullen J. A. (2007), « Modern pollen-vegetation relationships in subarctic Southern Greenland and the interpretation of fossil pollen data from the Norse landnám ", Journal of Biogeography, $\mathrm{n}^{\circ}$ 34, p. 473-488.

Schofield J. E., Edwards K. J., Christensen C. (2008), « Environmental impacts around the time of Norse landnám in the Qorlortoq valley, Eastern Settlement, Greenland ", Journal of Archaeological Science, $\mathrm{n}^{\circ}$ 35, p. 1643-1657.

van Geel B. (2001), « Non-pollen palynomorphs », in SMOL J. P., BiRKS H. J. B., LAST W. M. (dir.), Tracking Environmental Change Using Lake Sediments : Terrestrial, Algal and Silicaceous Indicators, vol. 3, Kluwer Academic Publishers, Dordrecht, p. 99-119.

VAN Geel B., AITROOT A. (2006), "Fossil ascomycetes in Quaternary deposits ", Nova Hedwigia, $\mathrm{n}^{\circ}$ 82, 3, 4, p. 313-329.

Vinther B. M., Jones P. D., Briffa K. R., Clausen H. B., Andersen K. K., DAHL-JENSEN D., JOHNSEN S. J. (2010), "Climatic signals in multiple highly resolved stable isotope records from Greenland ", Quaternary Science Reviews, $\mathrm{n}^{\circ} 29$, p. 522-538. 